\title{
Estrogen contributes to regulating iron metabolism through governing ferroportin signaling via an estrogen response element
}

\author{
Yi Qian ${ }^{\text {a,b }}$, Chunyang Yin a , Yue Chen ${ }^{\text {a,c }}$, Shuping Zhang ${ }^{\text {a,d }}$, Li Jiang ${ }^{\text {e }}$, Fudi Wang ${ }^{\text {, }}$, Meirong Zhao ${ }^{\text {b,* }}$, Sijin Liu ${ }^{\text {a,* }}$ \\ a State Key Laboratory of Environmental Chemistry and Ecotoxicology, Research Center for Eco-Environmental Sciences, Chinese Academy of Sciences, Beijing 100085, China \\ ${ }^{\mathrm{b}}$ College of Biological and Environmental Engineering, Zhejiang University of Technology, Hangzhou 310032, China \\ c Department of Urology, The Second Hospital of Tianjin Medical University, Tianjin Institute of Urology, Tianjin 300211, China \\ d Institute for Medical Engineering and Science, Massachusetts Institute of Technology, Cambridge, MA 02139, USA

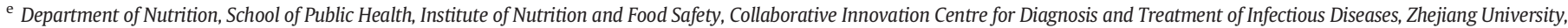 \\ Hangzhou, Zhejiang 310027, China
}

\section{A R T I C L E I N F O}

\section{Article history:}

Received 15 January 2015

Accepted 31 January 2015

Available online 4 February 2015

\section{Keywords:}

Estrogen

Ferroportin

Iron

Ovariectomy

Estrogen response element

\begin{abstract}
A B S T R A C T
Ferroportin (FPN) is the only known iron exporter in mammalian cells, and is universally expressed in most types of cells. FPN signaling plays a crucial role in maintaining iron homeostasis through governing the level of intracellular iron. Serum iron storage is conversely related with the estrogen level in the female bodies, and women in post-menopause are possibly subjected to iron retention. However, the potential effects of estrogen on iron metabolism are not clearly understood. Here, FPN mRNA transcription in all selected estrogen receptor positive $(E R+)$ cells was significantly reduced upon 17ß-estradiol (E2) treatment; and this inhibitory effect could be attenuated by ER antagonist tamoxifen. Likewise, in murine bone marrow-derived macrophages (BMDMs), FPN reduction with elevated intracellular iron (reflected by increased ferritin) was observed in response to E2; however, ferritin level barely responded to E2 in FPN-null BMDMs. The observation of inhibition of FPN mRNA expression was not replicated in $\mathrm{ER}^{-}$cells upon E2. A functional estrogen response element (ERE) was identified within the promoter of FPN, and this ERE was responsible for the suppressive effect of E2 on FPN expression. Moreover, ovariectomized (OVX) and sham-operated (SHAM) mice were used to further confirm the in vitro finding. The expression of hepatic FPN was induced in OVX mice, compared to that in the SHAM mice. Taken together, our results demonstrated that estrogen is involved in regulating FPN expression through a functional ERE on its promoter, providing additional insights into a vital role of estrogen in iron metabolism.
\end{abstract}

(c) 2015 Elsevier Inc. All rights reserved.

\section{Introduction}

Iron is an essential element to all forms of life. Iron is a necessary component for a large array of proteins involved in diverse functions including oxygen delivery, electron transfer, energy metabolism, transcriptional regulation and DNA replication [1-3]. It is considerably important to maintain a fine-tuned balance of iron metabolism. In fact, iron disorders in the form of either iron deficiency or overload cause detrimental impacts on cells [3-5]. Mammalian iron homeostasis is concertedly regulated through the hepcidin-ferroportin (FPN) axis that fundamentally governs iron absorption, transport, storage and utilization $[3,6]$. Iron needs the sole known iron exporter, FPN, to shuttle out of the cells.[7] As a transmembrane transporter, FPN is responsible for dietary iron absorption in enterocytes of small intestine, and also controls iron release from macrophages and other cells [8,9]. High levels of FPN lead to enforced dietary iron absorption and egress from storage

\footnotetext{
* Corresponding authors at: 18 Shuangqing Rd, Haidian, Beijing 100085, China. Tel./ fax: +861062849330 .

E-mail addresses: zhaomr@zjut.edu.cn (M. Zhao), sjliu@rcees.ac.cn (S. Liu).
}

out of cells including macrophages and hepatocytes, which is associated with several types of diseases, such as hereditary hemochromatosis and $\beta$-thalassemia caused by iron overload [5]. Inversely, FPN deficiency or loss of iron-exporting function could also result in problematic iron metabolism, namely iron retention in enterocytes and macrophages coupled to low plasma iron concentration $[3,10,11]$. Patients with this disease are characterized by low plasma transferrin saturation and iron retention predominantly in macrophages [5].

In light of FPN regulation, FPN protein concentration is prominently modulated through a posttranscriptional mechanism by the iron responsive element/iron regulatory protein (IRE/IRP) signaling, as iron abundance enforces FPN translation and iron deficit represses FPN translation [12-15]. Additionally, FPN could also be regulated by hypoxia inducible factor 2 (HIF2 $\alpha$ ), nuclear factor erythroid (Nrf2), and metalregulatory transcription factor 1 (MTF-1) at the transcriptional level in macrophages $[11,12,16]$. However, little is known about the regulation of FPN under different physiological settings including natural alterations of endogenous steroid hormones, such as estrogen.

Estrogen is an indispensable hormone implicated in a wide spectrum of physiological functions for organs including breast, bone and 
skin, especially in females [17-19]. Within the life span of a woman, the endogenous level of estrogen greatly fluctuates, particularly after menopause [20]. The pronounced manifestation of menopause is the cessation of menstruation, accompanied by estrogen deficiency and reduced functions of the ovaries [17]. A few previous studies have suggested the consequential change of iron levels after menopause [20,21]. Considering the change of iron, several reasons should be taken into account. For example, reduction of monthly blood loss would certainly help to retain body iron [17]. Recent studies from our research group and another group have uncovered the regulation of hepcidin by estrogen, linking estrogen to iron homeostasis $[22,23]$. Nonetheless, the impact of estrogen on iron metabolism is still elusive, and specifically, no insight has been gained for the potential regulation of estrogen on FPN.

In the current study, in an effort to determine the contribution of estrogen to FPN regulation, we employed a panel of in vitro and in vivo strategies. Overall, we deciphered a novel mechanism underlying the regulation of FPN by estrogen. Estrogen (17 $\beta$-estradiol, a primary endogenous female sex hormone) could significantly inhibit the mRNA transcription of FPN through a functional estrogen response element (ERE) within its promoter. Cells with FPN deficiency (namely FPN knockout cells) were resistant to estrogen-induced iron alterations through FPN. Consistent with the in vitro findings, FPN expression was elevated upon estrogen deficiency in mice with ovariectomy. Our combined data highlighted a crucial role for estrogen in iron metabolism through repressing FPN expression via an ERE within its promoter.

\section{Materials and methods}

\subsection{Chemicals and reagents}

17ß-Estradiol and tamoxifen were purchased from Sigma-Aldrich Co. Dimethyl sulfoxide (DMSO) (Solarbio Inc., China) was used to dissolve $17 \beta$-estradiol and tamoxifen. The final concentration of DMSO in culture medium was $<0.1 \%$.

\subsection{Cell culture}

Human acute monocytic leukemia cell line THP-1, human hepatic carcinoma cell lines SMMC-7721 and HepG2, and human breast cancer cell lines T47D and MDA-MB-231 were purchased from the Shanghai Cell Bank of Type Culture Collection of Chinese Academy of Science. All cells were cultured in medium with $5 \%$ charcoal-treated FBS (Hyclone) at $37{ }^{\circ} \mathrm{C}$ with $5 \% \mathrm{CO}_{2}$. To initiate differentiation, THP-1 cells were treated with $200 \mathrm{nM}$ Phorbol-12-myristate-13-acetate (PMA) for $24 \mathrm{~h}$, as previously described [24]. Thereafter, the medium containing PMA was replaced with fresh medium for further experiments. Murine bone marrow-derived macrophages (BMDMs) were isolated and cultured as previously described [25].

\subsection{Animal experiments}

All animal care and surgical procedures followed the guidelines of Animal Ethics Committee at the Research Center for Eco-environmental Sciences, Chinese Academy of Sciences. For animals, 8-week-old female C57BL/6 mice were purchased from the Vital River Laboratories (Beijing, China), and FPN1-floxed and LysM-Cre mice with the 129/SvEvTac background were provided by Dr. Fudi Wang [6,26-28]. LysM-Cre mice were mated with FPN $1^{\text {flox/flox }}$ mice to create the strain in which FPN was inactivated in macrophages. Mice were housed under specific pathogen-free and sterile conditions.

C57BL/6 mice were randomly divided into 2 groups of seven $(n=7)$. One group was bilaterally ovariectomized (OVX), and the mice in the other group were sham-operated following a surgical protocol previously described [29]. After 8 weeks, these mice were sacrificed. The liver, spleen, uterus and blood specimens from each mouse were collected for further experiments.

\subsection{Iron measurements}

Serum iron was measured with a kit on the basis of the manufacturer's instructions (Nanjing Jiancheng Bioengineering Institute). Liver and spleen iron contents were determined as previously described [25].

\subsection{Cell proliferation assay}

Cell growth was measured by Alamar blue and BrdU incorporation assays (Roche). Briefly, PMA-differentiated THP-1 cells were starved in serum-free medium overnight and then seeded at a density of $1.0 \times 10^{4}$ cells/well in 96-well plates with or without $1 \times 10^{-7} \mathrm{M} \mathrm{E2}$ for $24 \mathrm{~h}$. Thereafter, resazurin (Sigma) was added into each well at a final concentration of $10 \%$, followed by incubation for additional $2 \mathrm{~h}$ at $37{ }^{\circ} \mathrm{C}$. Finally, fluorescence intensity was recorded at an excitation wavelength of $530 \mathrm{~nm}$ with an emission wavelength of $590 \mathrm{~nm}$. With respect to the BrdU assay, we followed the standard instructions provided by the manufacturer.

\subsection{Real-time RT-PCR analysis}

Total RNAs from cells or liver tissues were purified with Trizol reagent following the manufacturer's instructions (Invitrogen). Liver tissues were first grinded in liquid nitrogen before total RNAs were extracted. Gene expression was measured using SYBR Green qPCR master mix (Promega) on a qRT-PCR machine (Bio-Rad). Primer sequences were as follows. Human FPN: forward, 5'-CTACTTGGGGAGATCGGA TGT-3', reverse, 5'-CTGGGCCACTTTAAGTCTAGC-3'; human CXCR4, forward, 5'-TCATCACGCTTCCCTTCTGG-3', reverse, 5'-CATGGACTGCCTTG CATAGGA-3'; human IL-6, forward 5'-CCAGAGCTGTGCAGATGAGT-3', reverse, 5'-CTGCAGCCACTGGTTCTGT-3'; human IL-1 $\beta$, forward, 5'-CTGTCC TGCGTGTTGAAAGA-3', reverse, 5'-TTGGGTAATTTTTGGGATCTACA-3'; human GAPDH, forward 5'-GAAGGTGAAGGTCGGAGT-3', reverse, 5'GAAGATGGTGATGGGATTTC-3'; mouse FPN, forward, 5'-GTCGGCCAGA TTATGACATTTG-3', reverse, 5'-ATTCCAACCGGAAATAAAACCA-3'; mouse GAPDH, forward, 5'-AAGGTCATCCCAGAGCTG-3', reverse, 5'GCCATGAGGTCCACCACCCT-3'. GAPDH was used as a loading control.

\subsection{Western blotting}

Cells were washed with ice cold PBS and then lysed in RIPA lysis buffer (Solarbio, China) supplemented with protease inhibitor cocktail (Roche), as previously described [30]. An equal amount of each lysate (80-100 $\mu \mathrm{g} / \mathrm{sample}$ ) was subjected to $10 \%$ sodium dodecyl sulfatepolyacrylamide gel electrophoresis (SDS-PAGE) and transferred to nitrocellulose membranes afterwards. Antibodies were against GAPDH (1:1000, Santa Cruz Biotechnology), FPN (1:500, Sigma), and ferritin light chain (1:1000, Abcam).

\subsection{Vector construction of FPN promoter with predicted ERE}

We identified a potential ERE (5'-TGTCAGTCTGGCC-3', $-376 /-388 \mathrm{bp}$ ) in the promoter of the human FPN gene using Dragon ERE Finder version 3.0 [31]. Two DNA fragments of the FPN promoter, one with the predicted ERE ( $-376 /-388 \mathrm{bp}$ ) and the other with deletion of the ERE, were inserted into the pGL3-promoter luciferase reporter vector (Promega). The vector with the predicted ERE was named as Pro-ERE, and the one without was named as Pro-ERE $\Delta$. These two plasmids were confirmed by DNA sequencing (Invitrogen).

\subsection{Luciferase assays}

Cells were seeded at a concentration of $1 \times 10^{5}$ cells $/ \mathrm{ml}$ in 24 -well plates. Cells were then co-transfected with $0.8 \mu$ g target plasmids (Pro-ERE or Pro-ERE $\Delta$ ) and 80 ng Renilla luciferase plasmid by 
Lipofectamine 2000 (Invitrogen) following the manufacturer's instructions. After $4 \mathrm{~h}$ incubation, the culture medium was replaced with phenol red-free medium with $5 \%$ charcoal-treated FBS, and thereafter $1 \times 10^{-7} \mathrm{M}$ E2 was added for $24 \mathrm{~h}$. Cells were then lysed with $100 \mu \mathrm{l}$ lysis buffer after washing with PBS, and cellular extracts were subsequently examined for luciferase activity using the dual-luciferase reporter assay system (Promega). The relative luciferase activities were normalized to those of Renilla luciferase.

\subsection{Image quantification of Western blots}

The intensity of autoradiogram in Western blotting was quantified using the software ImageJ (NIH, http://rsbweb.nih.gov). Quantified results for each protein were normalized to those of the loading control.

\subsection{Statistical analysis}

Independent $t$ test or One-Way ANOVA test was employed to analyze the experimental data. Data were shown in mean \pm standard error. Statistical significance was determined with $P$ value less than 0.05 .

\section{Results}

\subsection{E2 repressed the expression of FPN in PMA-differentiated THP-1 cells}

The ER $\alpha$-positive THP- 1 cells are widely used as an ideal model in studying functions of macrophages, such inflammation [32]. THP-1 cells were also used to study iron metabolism in macrophages $[3,33]$. Here, to determine the potential role of $\mathrm{E} 2$ in regulating the expression of FPN, we first measured the mRNA and protein levels of FPN upon $1 \times 10^{-7}$ M E2 treatment in PMA-differentiated THP-1 cells for $24 \mathrm{~h}$. E2 treatment per se did not undermine the cell viability, as evidenced by the viability assay and the BrdU incorporation assay (Fig. 1A \& B). The FPN mRNA level was reduced by approximately $30 \%$ in response to E2 (Fig. $2 \mathrm{~A}, \mathrm{P}<0.05$ ), in analogy to the alteration of the established estrogen down-regulated gene chemokine receptor type 4 (CXCR4) [34] upon E2 (Fig. 2A). Consistently, E2 also reduced FPN concentration by around $30 \%$ at the protein level (Fig. 2B), suggesting that E2 could significantly repress FPN expression. Moreover, upon pre-treatment with $40 \mu \mathrm{g} / \mathrm{ml}$ cycloheximide (CHX), an inhibitor of protein biosynthesis in eukaryotic organisms, the FPN mRNA level was still reduced by $25 \%$ in response to E2 (Fig. 2C), suggesting that the suppressive effect of E2 on FPN was through a transcriptional mechanism, rather than a translational mechanism. Additionally, tamoxifen, which acts as an antagonist of E2 action by binding to ER, [35] notably reversed this inhibitory effect at the concentration of $1 \mu \mathrm{M}$ (Fig. 2D), further indicating that FPN could be modulated through E2 signaling pathway.

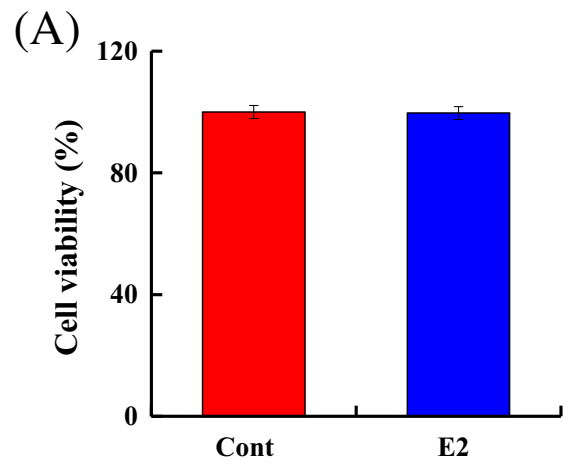

Due to the reduction of FPN concentration, ferritin, an iron storage protein, was increased by more than 50\% (Fig. 2B). Previous studies have suggested that the increased cellular iron was highly associated with reduced production of inflammatory cytokines from macrophages, including interleukin-6 (IL-6) and IL-1 $\beta$ [36-38]. Therefore, we hypothesized that E2-induced reduction of FPN could likely in turn undermine the inflammatory responses of THP- 1 cells. To test this hypothesis, we detected the mRNA levels of IL- 6 and IL-1 $\beta$ upon treatment of E2 or ferric nitrilotriacetate (Fe-NTA at $100 \mu \mathrm{M}$ ) as a positive control. As a result, the expression levels of IL- 6 and IL- $1 \beta$ were impaired coupled to elevated intracellular iron content, due to the reduction of FPN concentration (Fig. 3A \& B), analogous to a previous study [36]. These data suggested that E2 could remarkably down-regulate FPN at the transcriptional level in human macrophages, leading to elevated intracellular iron levels and weakened inflammatory responses.

\subsection{Confirmation of E2-mediated transcriptional regulation of FPN}

To validate the regulation of FPN expression by estrogen, we further examined this mechanism in different types of cells. Hepatocytes have relatively high level of FPN on the plasma membrane [39]. Representative cell models of hepatocytes, such as HepG2 and SMMC-7721 cells, were conventionally used in estrogen-related studies [22,23]. Thus, we looked into the alteration of FPN expression upon E2 in HepG2 and SMMC-7721 cells. In parallel to the alterations in THP-1 cells upon E2 (Fig. 2A), the expression of FPN was also reduced in both HepG2 and SMMC-7721 cells by $30 \%$ and $40 \%$ at the mRNA level, respectively (Fig. 4A \& B, P < 0.05). Similar alterations of FPN at the protein level were demonstrated (Fig. 4A \& B). In support of the regulation of FPN by estrogen, CXCR4 expression was similarly restrained in both HepG2 and SMMC-7721 cells by approximately 50\% (Fig. 4A \& B, P < 0.05 ).

In an attempt to confirm the finding of $\mathrm{E} 2$ in governing FPN expression, we additionally chose two human breast cancer cell lines: T47D and MDA-MB-231 cells, which were ER $\alpha$-positive and ER $\alpha$-negative, respectively [40,41]. As illustrated in Fig. 4C, both the mRNA and protein levels of FPN were reduced by over $20 \%$ in T47D cells in the presence of E2. However, FPN expression did not respond to E2 in MDA-MB-231 cells (Fig. 4D). Therefore, these results together indicated that E2 played a role in repressing FPN expression in ER-positive cells.

\subsection{E2 inhibited FPN transcription in BMDMs}

Next, we sought to investigate whether E2 induced similar influence on murine macrophages. Thus, primary wild type (WT) BMDMs from mice with the 129/SvEvTac background were cultured, and then treated with or without $1 \times 10^{-7} \mathrm{M} \mathrm{E} 2$ for $24 \mathrm{~h}$. Consistent with the results in human cell lines, WT BMDMs revealed nearly $50 \%$ reduction of FPN at both mRNA and protein levels upon E2 (Fig. 5A, $\mathrm{P}<0.05$ ). As a result of FPN reduction, the iron storage protein ferritin was elevated by $30 \%$

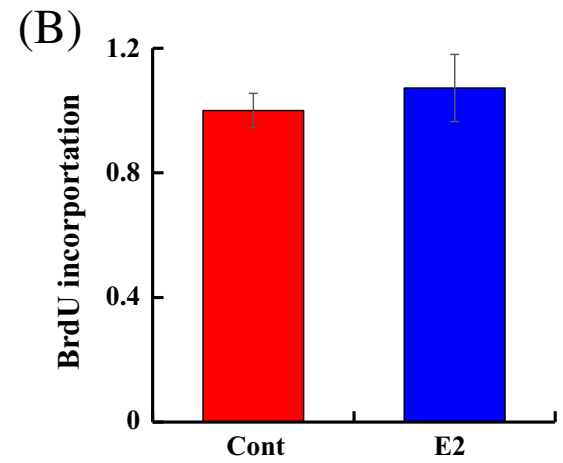

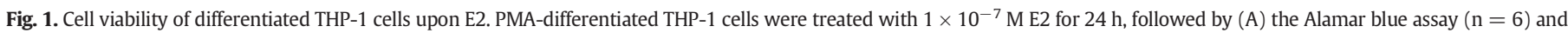
(B) the BrdU assay $(\mathrm{n}=6)$. 
(A)

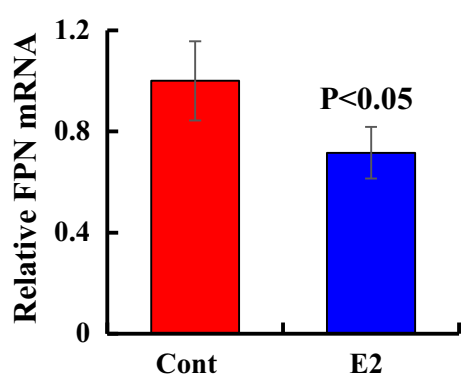

(B)

$1.00 \quad 0.64$

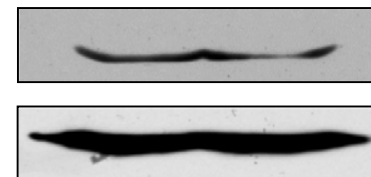

FPN

1.00

1.80

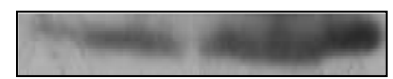

Ferritin

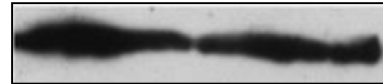

GAPDH

GAPDH

Cont

E2

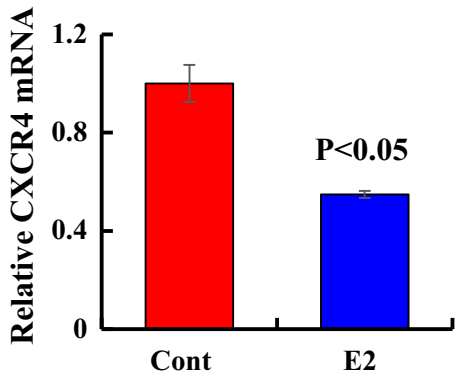

(C)

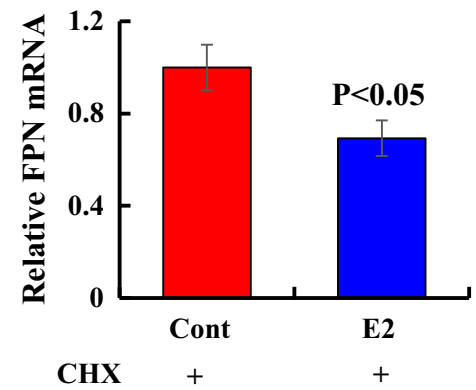

(D)

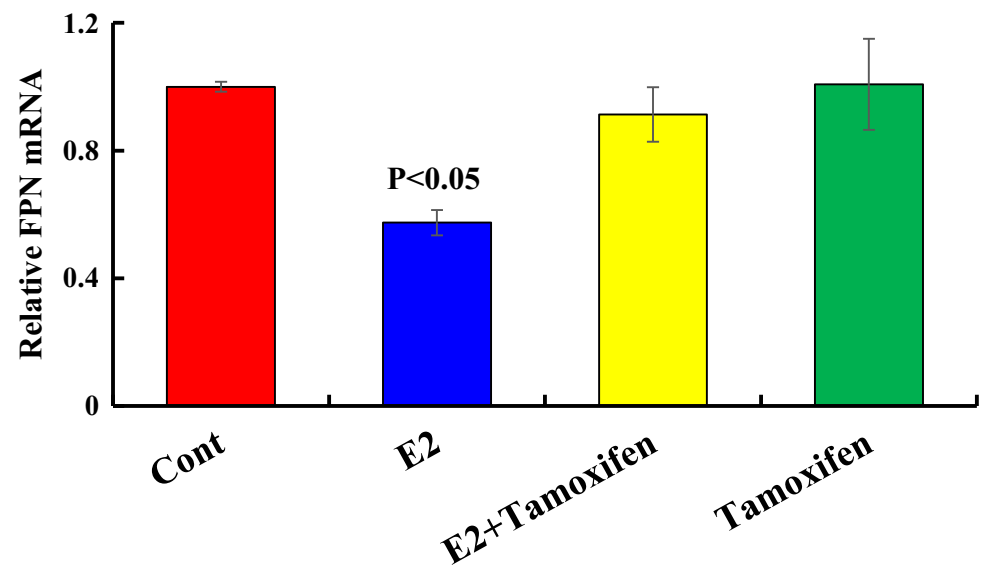

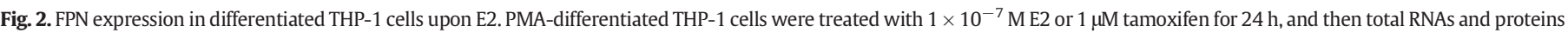

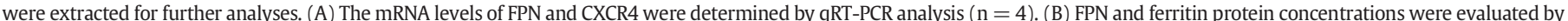

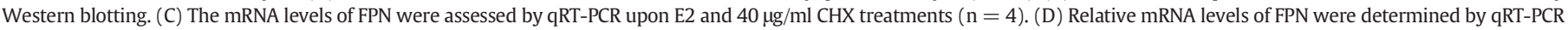
upon exposure to E2 and/or $1 \mu \mathrm{M}$ tamoxifen $(\mathrm{n}=4)$.

(Fig. 5B), indicating that estrogen could substantially alter iron status in WT BMDMs through down-regulating FPN expression. In an effort to further confirm the finding of FPN reduction induced by E2 on murine macrophages, FPN deficient BMDMs were employed according to the strategy described by Zhang et al. [28] FPN mRNA expression was decreased by nearly $90 \%$ in FPN-null BMDMs (Fig. 6A), compared to the WT cells. Moreover, the protein level of FPN was almost undetectable (Fig. 6B), suggesting that FPN was mostly ablated in these BMDMs. Under the setting of FPN deficiency, we demonstrated that the ferritin protein level was no longer responding to E2 levels (Fig. 6C). These data collectively demonstrated that E2 acted to restrain the transcription of FPN, and in turn caused intracellular iron retention in mouse macrophages.
3.4. FPN transcription was regulated by E2 through a functional ERE within the FPN promoter

Furthermore, we strived to decipher the molecular mechanisms responsible for the inhibition of FPN transcription upon E2. Hou and colleagues from our research group previously identified an ERE within the promoter of hepcidin, and this ERE was responsible for E2-induced suppression of hepcidin expression in hepatocytes [23]. Therefore, we assumed that the promoter of FPN might also harbor a similar estrogen response site. It has been recognized that the conserved sequence of canonical ERE consists of palindromic half-sites interspaced by three nucleotides, namely, 5'-GGTCA-NNN-TGACC-3' [42]. However, other non-canonical EREs possibly have one or more base aberrations from 

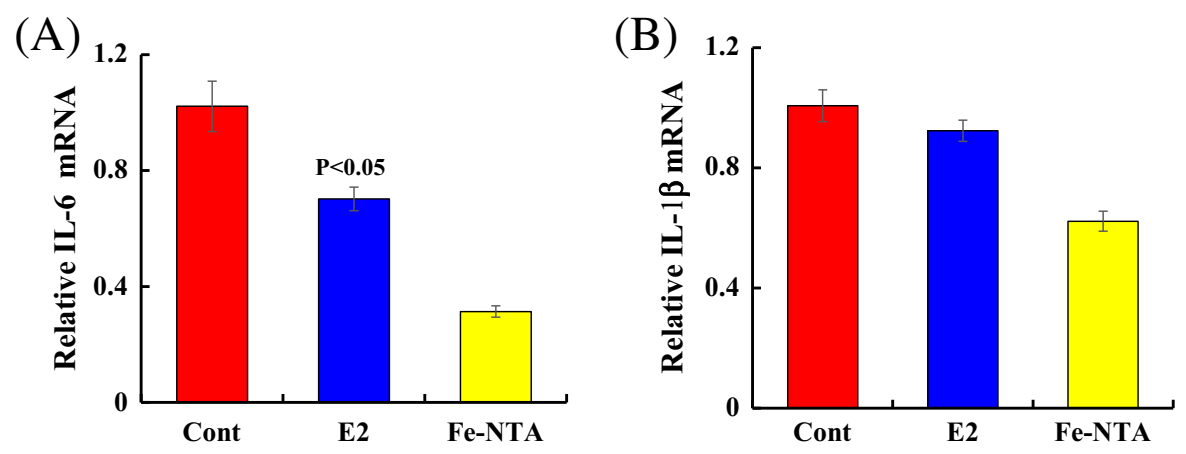

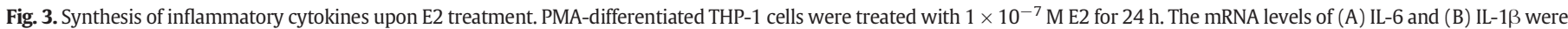
assayed $(n=4)$. Fe-NTA at $100 \mu \mathrm{M}$ was used here as a control to mimic excess iron.

(A)

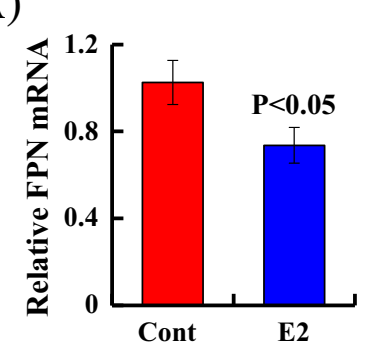

(B)

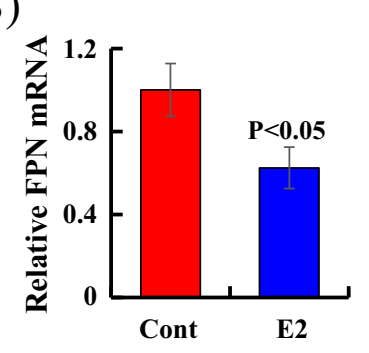

(C)

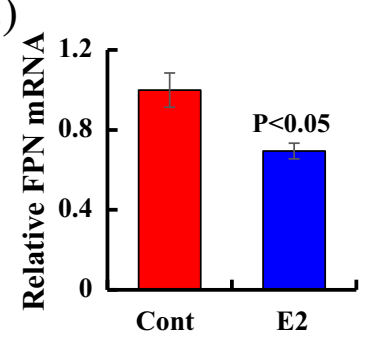

(D)

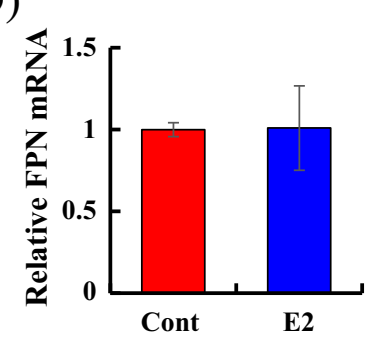

SMMC-7721 cells
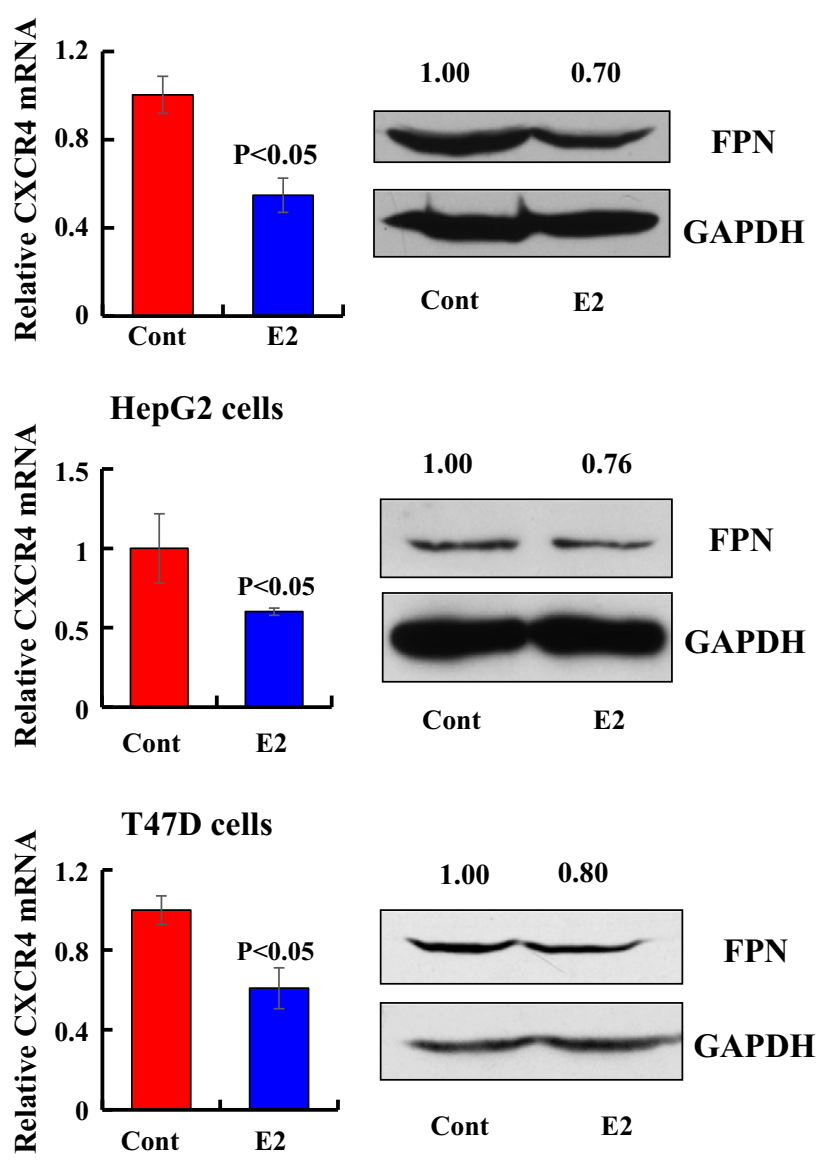

MDA-MB-231 cells

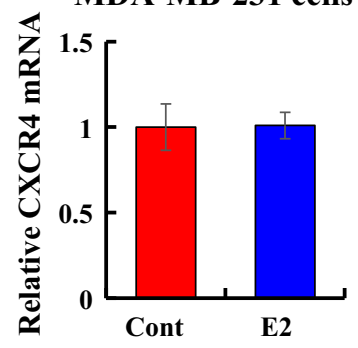

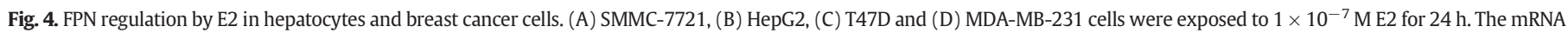
levels of FPN and CXCR4 ( $n=4)$, and the FPN protein concentrations were then determined, respectively. 

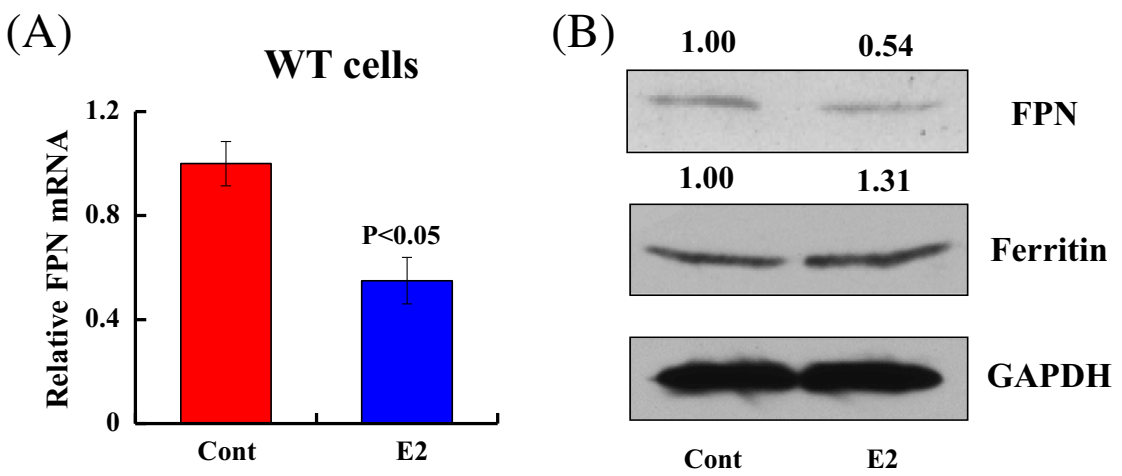

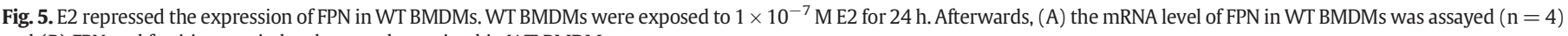
and (B) FPN and ferritin protein levels were determined in WT BMDMs.

this sequence [43]. We found a putative ERE (5'-TGTCAGTCTGGCC-3', $-376 /-388 \mathrm{bp}$ ) in the promoter region of the FPN gene with the software Dragon ERE Finder version 3.0, as previously reported [31] (Fig. 7A). In order to define the function of this predicted ERE, we constructed two reporter plasmids, i.e. one with intact ERE (Pro-ERE) and one with ERE deletion (Pro-ERE $\Delta$ ). As shown in Fig. 7B, the transcriptional activity of Pro-ERE was reduced by 20\% upon E2 treatment, whereas the response to E2 was thoroughly lost in Pro-ERE $\Delta$ transfected cells, similar to the mechanism of E2-mediated repression of hepcidin, in our previous study [23]. These findings therefore suggested that the putative ERE ( $-376 /-388 \mathrm{bp})$ functionally responded to E2. In other words, the transcription of FPN was inhibited through the action of E2 on the ERE in the FPN promoter region.

\subsection{FPN expression was elevated in estrogen-deficient mice}

In an attempt to elucidate the biological impact of FPN repression caused by estrogen in animals, OVX mice were chosen as an experimental model. First, uterus alteration of the mice with or without ovariectomy was investigated. A significant decline in the uterus weight (by 60\%) was observed in the OVX group after 8 weeks, compared to the SHAM control group (Fig. 8A, $\mathrm{P}<0.05$ ), suggesting that estrogen was successfully suppressed in OVX mice. Meanwhile, the mRNA level of hepatic FPN was measured. An increase by approximately $50 \%$ was demonstrated in the OVX mice relative to the SHAM mice (Fig. 8B, $\mathrm{P}<0.05$ ), further supporting the regulation of FPN by estrogen. Additionally, serum iron was slightly reduced associated with mildly increase of hepatic and splenic iron (Fig. 8C). As our previous study showed that hepcidin could also be down-regulated by E2 [23], we subsequently inspected the hepcidin expression. In agreement with the previous finding, the absence of estrogen greatly enhanced the mRNA level of hepcidin by nearly 2 fold (Fig. 8D, $\mathrm{P}<0.05$ ), suggesting that hepcidin was elevated to a greater extent than FPN. The induction of hepcidin counteracted the increase of FPN in response to estrogen deficiency, leading to the compensation of iron status. Thus, the iron mass in serum, spleen and liver was not dramatically changed when hepcidin and FPN were simultaneously altered upon estrogen loss in the OVX mice. Nonetheless, these data verified the regulation of FPN expression by estrogen in animals.

\section{Discussion}

Iron is an essential bio-metal for almost all types of organisms, as it is a component of iron-sulfur cluster-containing proteins, including DNA helicase enzymes involved in DNA replication and other factors implicated in electron transport and oxidative phosphorylation [44-46]. Thus, iron is necessary for cellular growth and other biological functions that closely depend on energy metabolism, DNA replication, and heme synthesis [47]. Systemic iron homeostasis is strictly fine-tuned through various mechanisms, of which the hepcidin-FPN axis fundamentally governs the global iron absorption, distribution, utilization, and egress [48]. However, disordered iron metabolism would likely give rise to a large array of dysfunctions, morbidities and even diseases, such as cancers [5,49-53]. In fact, a myriad of epidemiological studies have documented a considerable contribution of elevated body iron to cancer

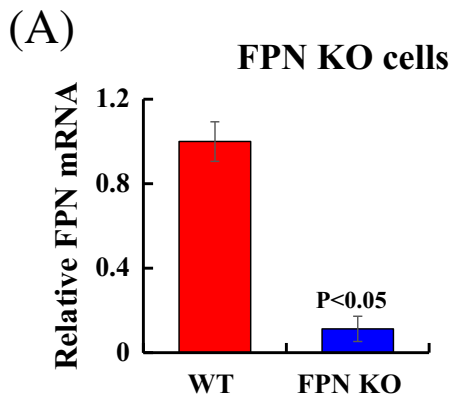

(B)

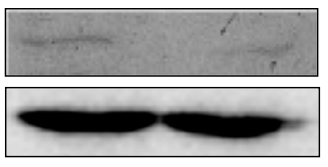

\section{FPN}

GAPDH
(C)

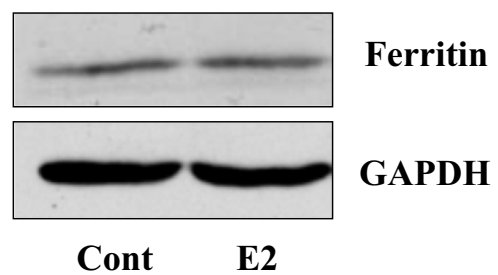

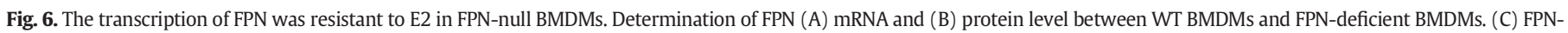
deficient BMDMs were treated with $1 \times 10^{-7} \mathrm{M} \mathrm{E2}$ for $24 \mathrm{~h}$, and then the protein concentration of ferritin was assessed through Western blotting. 
(A)

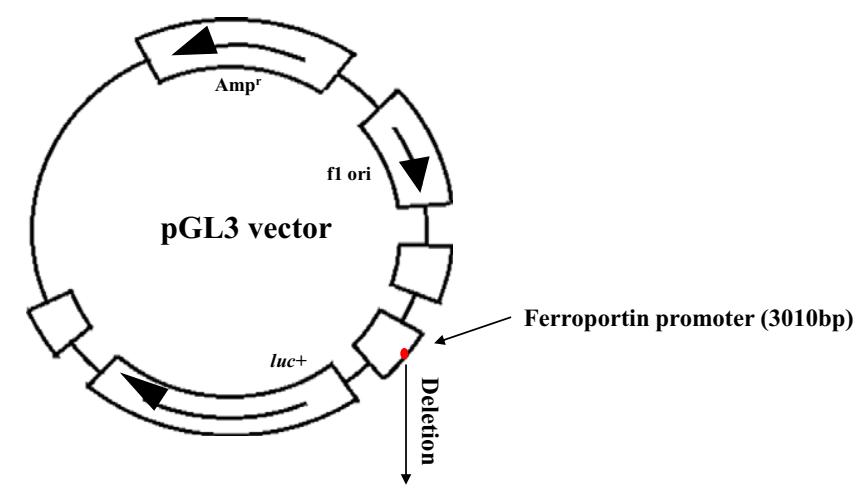

ACTGGGTGTCAGTCTGGCCTGTGCT (-370/-394bp)
(B)
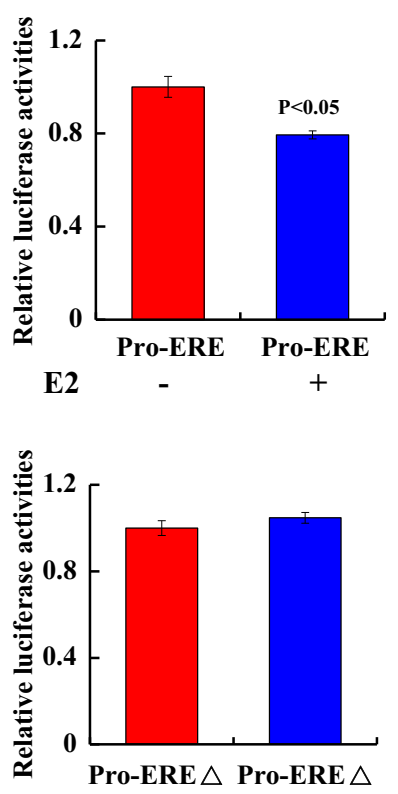

E2

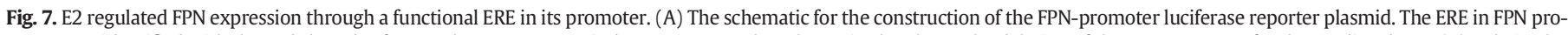

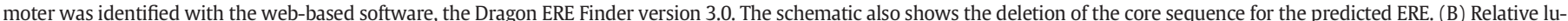

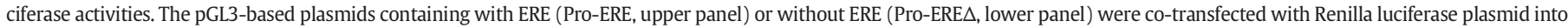
HepG2 cells. The relative firefly luciferase activities were normalized to those of the Renilla luciferase.

risk of occurrence and prognosis [52-57]. In stark contrast to normal cells, iron demand is remarkably enforced in cancer cells to meet the enhanced cellular activities including DNA synthesis and energy generation $[58,59]$. The reasons for elevated body iron burden are poorly understood in cancer patients. In light of iron metabolism in women, menopause and endogenous estrogen alteration have been postulated to physiologically account for the change of body iron mass $[17,60]$.

Estrogen is a hormone that physiologically dictates the integrity and functions for a variety of cells. The endogenous estrogen level fluctuates before menopause, and drops drastically post menopause. Reduced estrogen in post-menopausal women is associated with a wide spectrum of symptoms, such as bone loss, sleep disorders, and weight gain and

(A)

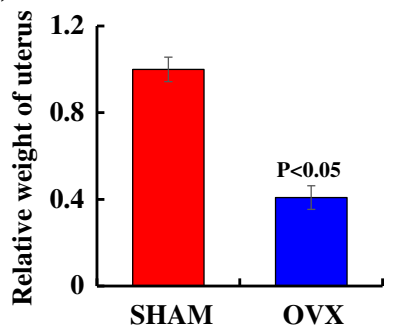

(C)
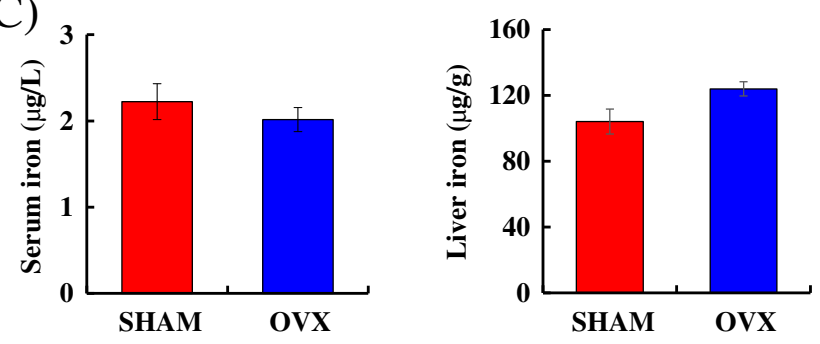

depression, resulting in increased risk of osteoporosis and other diseases. Elevated body iron has been documented in postmenopausal women for unclear reasons [20,52,61]. For example, a previous study identified a negative correlation between serum ferritin and estrogen level in women [17]. The mechanisms underlying the correlation between estrogen and iron metabolism is still elusive thus far. Here in the current study, we offered a line of direct evidence demonstrating the contribution of estrogen to the regulation of the iron exporter FPN concentration. We overall recognized the transcriptional repression of FPN expression by estrogen through a functional ERE in the promoter region of FPN gene. The current findings would be beneficial to explain increased body iron mass in post-menopausal women.

(B)

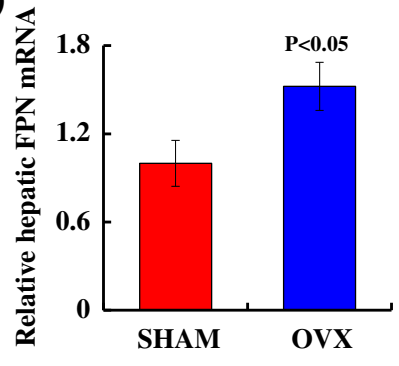

(D)
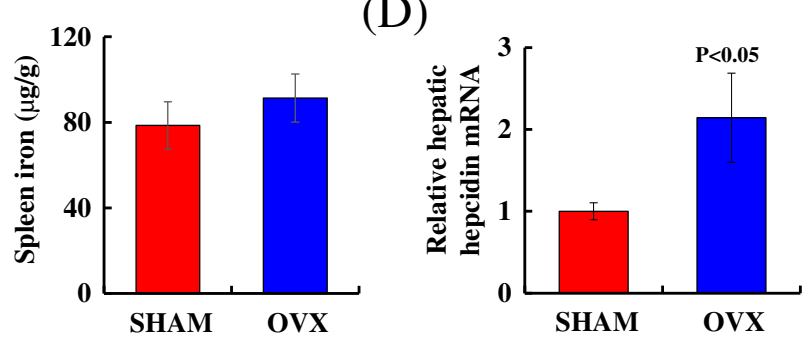

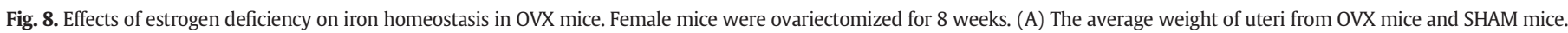
(B) Hepatic FPN mRNA level. (C) Iron mass in the serum, liver and spleen. (D) Hepatic hepcidin mRNA level for both OVX and SHAM groups ( $\mathrm{n}=7$ ). 


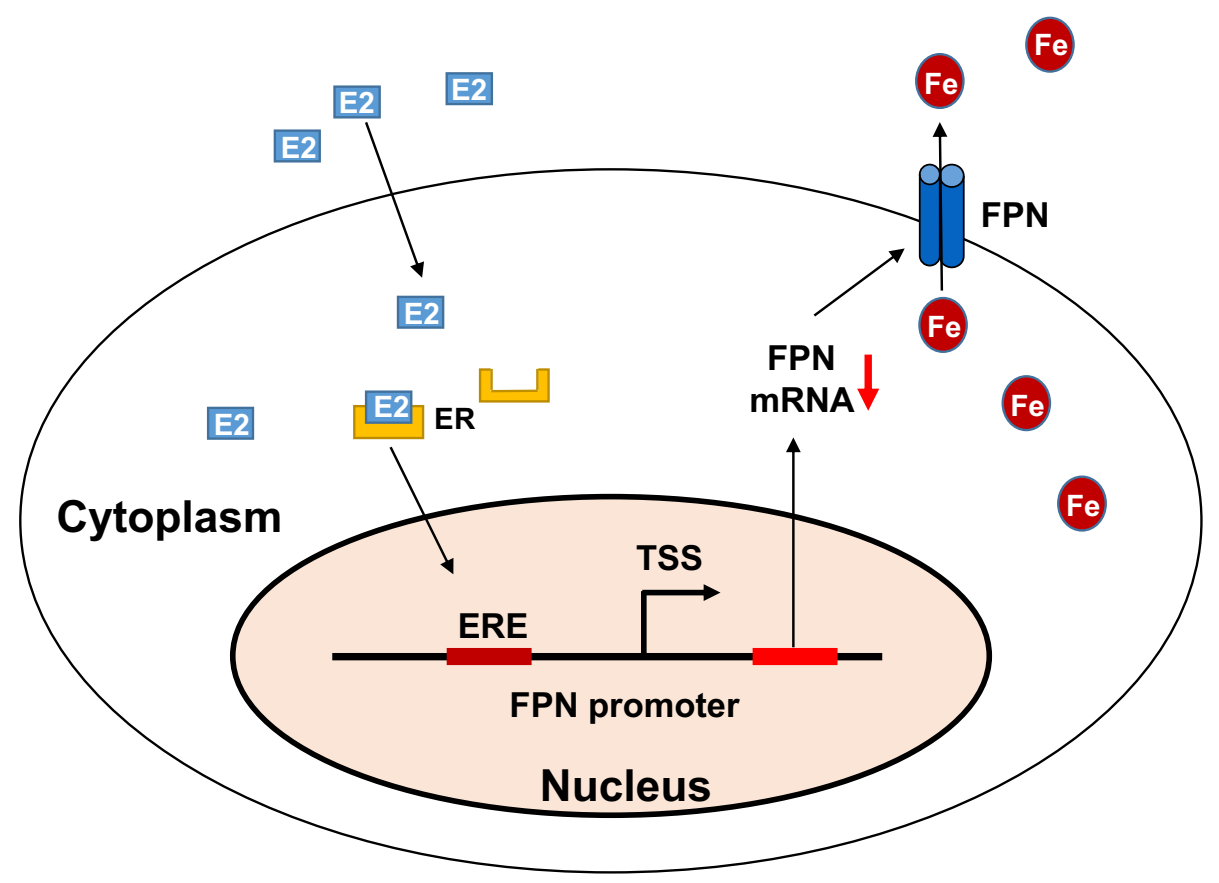

Fig. 9. A schematic delineating the role of estrogen in maintaining iron metabolism through regulating FPN transcription.

Our research group and another group have identified the regulation of hepcidin expression by estrogen $[22,23]$. Our previous data characterized an inhibitory effect of estrogen on hepcidin expression via a functional ERE within its promoter [23]. However, the ligand of hepcidin, FPN, has not been investigated in response to estrogen to date. FPN plays a crucial role in maintaining the concerted iron homeostasis through controlling iron egress, and dysregulation of FPN would give rise to disordered iron metabolism, [10] likely resulting in FPN diseases, such as hereditary hemochromatosis and $\beta$-thalassemia [5]. Additionally, we also recently found reduced level of FPN in breast cancers, associated with poor prognosis in breast cancer patients $[11,30]$. To keep the balance of systemic iron metabolism, FPN is predominantly regulated by hepcidin through binding and inducing ubiquitin-dependent proteasomal degradation of FPN protein [6]. FPN concentration is also subjected to the regulation by the iron responsive element (IRE)/iron responsive protein (IRP) system at the post-transcriptional level [15, $62,63]$. Moreover, emerging evidence suggests that FPN expression is also transcriptionally regulated. For example, we recently demonstrated that myeloid zinc-finger 1 (MZF-1) could directly transactivate FPN expression and then enhance the FPN-mediated iron export, resulting in a significant suppression of prostate tumor growth [11]. In the current study, we first demonstrated that estrogen treatment significantly reduced the expression of FPN in multiple human cell lines including macrophages, hepatocytes and ER positive breast cancer cells, coupled to augmented intracellular iron. Moreover, the repression of FPN expression by estrogen was not replicated in ER-negative cells. Tamoxifen, an ER antagonist, could reverse this repression. These data together suggested that estrogen-regulated FPN expression was dependent on ER. Analogously, the expression of FPN in WT BMDMs was greatly inhibited upon E2 activation, leading to elevated ferritin levels; however, FPNdeficient BMDMs were resistant to the repressive effect by estrogen. Moreover, we defined the molecular mechanism underlying estrogenconducted inhibition of FPN expression. Namely, a functional ERE within the promoter of FPN was responsible for the suppressive effect induced by E2. Our results offered an additional explanation for enhanced body iron storage in postmenopausal women [22]. In support of this statement, our results revealed that the expression of hepatic FPN was elevated in OVX mice, compared to SHAM mice. Meanwhile, hepatic hepcidin expression was also enforced in OVX mice, relative to
SHAM mice, indicating restrained iron absorption and iron egress in response to hepcidin-induced FPN degradation. In parallel to the findings from the current animal study, recent clinical investigations also identified increased serum hepcidin concentrations in postmenopausal women, relative to premenopausal women $[64,65]$. To this end, the induction of FPN was compensated by the increase of hepcidin in the absence of estrogen in mice. Thus, only mild alterations of serum iron and splenic and hepatic iron mass were observed in OVX mice, relative to SHAM mice. However, the relative contribution of estrogenregulated FPN and hepcidin to the net effects on systemic iron homeostasis is still not clear, and therefore, further detailed investigations are warranted.

\section{Conclusion}

To summarize, we deciphered a novel mechanism for estrogen in contributing to maintaining iron metabolism through regulating FPN signaling (Fig. 9). Estrogen exerts the transcriptional inhibitory effect on FPN expression via a functional ERE within its promoter region. This study provides additional explanation for body iron changes in women upon estrogen alterations, such as a dramatic decline in the estrogen level in postmenopausal women. Since increased serum iron and tissue iron retention were observed in postmenopausal women, [30,66] excess iron would likely incur in detrimental effects on health. Therefore, in addition to its pronounced role in ameliorating menopausal symptoms, administration of a proper dose of estrogen (namely estrogen replacement therapy) might help to orchestrate systemic iron homeostasis.

\section{Competing interests}

No potential conflicts of interest were disclosed by the authors. There are no financial competing interests to declare in relation to this manuscript.

\section{Acknowledgments}

This work was supported by a grant under the National "973" Program (grant number: 2014CB932000), the Strategic Priority Research 
Program of the Chinese Academy of Sciences (grant no. XDB14000000) and grants from the National Natural Science Foundation of China (grant numbers: 21425731, 21377159, 21177151, 21321004, 31030039, 31225013 and 31330036). We thank all the laboratory members for their great assistance with experiments and reagents.

\section{References}

[1] A. Sheftel, O. Stehling, R. Lill, Trends Endocrinol. Metab. 21 (2010) 302-314

[2] R. Gozzelino, V. Jeney, M.P. Soares, Annu. Rev. Pharmacol. Toxicol. 50 (2010) 323-354.

[3] R. Evstatiev, C. Gasche, Gut 61 (2012) 933-952.

[4] Q. Liu, L. Sun, Y. Tan, G. Wang, X. Lin, L. Cai, Curr. Med. Chem. 16 (2009) 113-129.

[5] T. Ganz, E. Nemeth, Annu. Rev. Med. 62 (2011) 347-360.

[6] A. Donovan, C.A. Lima, J.L. Pinkus, G.S. Pinkus, L.I. Zon, S. Robine, N.C. Andrews, Cell Metab. 1 (2005) 191-200.

[7] A.T. McKie, P. Marciani, A. Rolfs, K. Brennan, K. Wehr, D. Barrow, S. Miret, A. Bomford, T.J. Peters, F. Farzaneh, M.A. Hediger, M.W. Hentze, R.J. Simpson, Mol. Cell 5 (2000) 299-309.

[8] S. Abboud, J. Biol. Chem. 275 (2000) 19906-19912.

[9] A. Donovan, A. Brownlie, Y. Zhou, J. Shepard, S.J. Pratt, J. Moynihan, B.H. Paw, A. Drejer, B. Barut, A. Zapata, T.C. Law, C. Brugnara, S.E. Lux, G.S. Pinkus, J.L. Pinkus, P.D. Kingsley, J. Palis, M.D. Fleming, N.C. Andrews, L.I. Zon, Nature 403 (2000) 776-781.

[10] A. Pietrangelo, Blood Cells Mol. Dis. 32 (2004) 131-138.

[11] Y. Chen, Z. Zhang, K. Yang, J. Du, Y. Xu, S. Liu, Oncogene (2014). http://dx.doi.org/ 10.1038/onc.2014.310

[12] M. Taylor, A. Qu, E.R. Anderson, T. Matsubara, A. Martin, F.J. Gonzalez, Y.M. Shah, Gastroenterology 140 (2011) 2044-2055.

[13] D.M. Ward, J. Kaplan, Biochim. Biophys. Acta 2012 (1823) 1426-1433.

[14] M.D. Knutson, M.R. Vafa, D.J. Haile, M. Wessling-Resnick, Blood 102 (2003) 4191-4197.

[15] M.U. Muckenthaler, B. Galy, M.W. Hentze, Annu. Rev. Nutr. 28 (2008) 197-213.

[16] M.B. Troadec, D.M. Ward, E. Lo, J. Kaplan, I. De Domenico, Blood 116 (2010) 4657-4664.

[17] J. Jian, E. Pelle, X. Huang, Antioxid. Redox Signal. 11 (2009) 2939-2943.

[18] L.R. Nelson, S.E. Bulun, J. Am. Acad. Dermatol. 45 (2001) S116-S124.

[19] P.J. Simm, A. Bajpai, V.C. Russo, G.A. Werther, Pediatr. Endocrinol. Rev. 6 (2008) 32-41.

[20] L.R. Zacharski, D.L. Ornstein, S. Woloshin, L.M. Schwartz, Am. Heart J. 140 (2000) 98-104.

[21] S.M. Penckofer, K. Holm, D. Schwertz, P. Chandler, Womens Health Issues 7 (1997) 55-63.

[22] Y. Ikeda, S. Tajima, Y. Izawa-Ishizawa, Y. Kihira, K. Ishizawa, S. Tomita, K. Tsuchiya, T. Tamaki, PLoS ONE 7 (2012) e40465.

[23] Y. Hou, S. Zhang, L. Wang, J. Li, G. Qu, J. He, H. Rong, H. Ji, S. Liu, Gene 511 (2012) 398-403.

[24] J. Zhou, P. Zhu, J.L. Jiang, Q. Zhang, Z.B. Wu, X.Y. Yao, H. Tang, N. Lu, Y. Yang, Z.N. Chen, BMC Cell Biol. 6 (2005) 25.

[25] S. Liu, R.N. Suragani, F. Wang, A. Han, W. Zhao, N.C. Andrews, J.J. Chen, J. Clin. Invest. 117 (2007) 3296-3305.

[26] E. Schaller, A.J. Macfarlane, R.A. Rupec, S. Gordon, A.J. McKnight, K. Pfeffer, Mol. Cell. Biol. 22 (2002) 8035-8043.

[27] B.E. Clausen, C. Burkhardt, W. Reith, R. Renkawitz, I. Forster, Transgenic Res. 8 (1999) 265-277.

[28] Z. Zhang, F. Zhang, P. An, X. Guo, Y. Shen, Y. Tao, Q. Wu, Y. Zhang, Y. Yu, B. Ning, G. Nie, M.D. Knutson, G.J. Anderson, F. Wang, Blood 118 (2011) 1912-1922.

[29] R.L. Jilka, G. Hangoc, G. Girasole, G. Passeri, D.C. Williams, J.S. Abrams, B. Boyce, H. Broxmeyer, S.C. Manolagas, Science 257 (1992) 88-91.
[30] S. Zhang, Y. Chen, W. Guo, L. Yuan, D. Zhang, Y. Xu, E. Nemeth, T. Ganz, S. Liu, Cell. Signal. 26 (2014) 2539-2550.

[31] V.B. Bajic, S.L. Tan, A. Chong, S. Tang, A. Strom, J.A. Gustafsson, C.Y. Lin, E.T. Liu, Nucleic Acids Res. 31 (2003) 3605-3607.

[32] X. Li, M. Li, X. Bai, Arch. Biochem. Biophys. 549 (2014) 26-31.

[33] A. Cutone, A. Frioni, F. Berlutti, P. Valenti, G. Musci, M.C. Bonaccorsi di Patti, Biometals 27 (2014) 807-813.

[34] J. Frasor, J.M. Danes, B. Komm, K.C. Chang, C.R. Lyttle, B.S. Katzenellenbogen, Endocrinology 144 (2003) 4562-4574.

[35] H. Hao, M. d'Alincourt-Salazar, K.M. Kelley, A. Shatnawi, S. Mukherjee, Y.M. Shah, M. Ratnam, Oncogene 26 (2007) 7872-7884.

[36] H. Oexle, A. Kaser, J. Most, R. Bellmann-Weiler, E.R. Werner, G. Werner-Felmayer, G. Weiss, J. Leukoc. Biol. 74 (2003) 287-294.

[37] M. Nairz, G. Fritsche, M.L. Crouch, H.C. Barton, F.C. Fang, G. Weiss, Cell. Microbiol. 11 (2009) 1365-1381.

[38] A.R. O'Brien-Ladner, S.R. Nelson, W.J. Murphy, B.M. Blumer, L.J. Wesselius, Am. J Respir. Cell Mol. Biol. 23 (2000) 112-119.

[39] E. Nemeth, M.S. Tuttle, J. Powelson, M.B. Vaughn, A. Donovan, D.M. Ward, T. Ganz, J. Kaplan, Science 306 (2004) 2090-2093.

[40] S.Q. To, V. Cheung, K.A. Lazarus, K.C. Knower, C.D. Clyne, Mol. Cell. Endocrinol. 394 (2014) 21-28.

[41] R. Narayanan, S. Ahn, M.D. Cheney, M. Yepuru, D.D. Miller, M.S. Steiner, PLoS ONE 9 (2014) e103202.

[42] A. Maggi, A. Villa, J. Steroid Biochem. Mol. Biol. 139 (2014) 262-269.

[43] C.M. Klinge, Nucleic Acids Res. 29 (2001) 2905-2919.

[44] Y. Wu, R.M. Brosh Jr., Nucleic Acids Res. 40 (2012) 4247-4260.

[45] K. Brzoska, S. Meczynska, M. Kruszewski, Acta Biochim. Pol. 53 (2006) 685-691.

[46] T.A. Rouault, W.H. Tong, Nat. Rev. Mol. Cell Biol. 6 (2005) 345-351.

[47] M.W. Hentze, M.U. Muckenthaler, N.C. Andrews, Cell 117 (2004) 285-297.

[48] T. Ganz, Blood 117 (2011) 4425-4433.

[49] J. Cullis, Clin. Med. 13 (2013) 193-196

[50] R.G. Stevens, D.Y. Jones, M.S. Micozzi, P.R. Taylor, N. Engl. J. Med. 319 (1988) 1047-1052.

[51] A.J. Cross, R. Sinha, R.J. Wood, X. Xue, W.-Y. Huang, M. Yeager, R.B. Hayes, M.J. Gunter, Cancer Prev. Res. (Phila.) 4 (2011) 1465-1475.

[52] X. Huang, Lancet Oncol. 9 (2008) 803-807.

[53] M.K. Siddiqui, Singh S. Jyoti, P.K. Mehrotra, K. Singh, R. Sarangi, Environ. Int. 32 (2006) 630-637.

[54] P. Knekt, A. Reunanen, H. Takkunen, A. Aromaa, M. Heliövaara, T. Hakuunen, Int. J. Cancer 56 (1994) 379-382.

[55] R.G. Stevens, B.I. Graubard, M.S. Micozzi, K. Neriishi, B.S. Blumberg, Int. J. Cancer 56 (1994) 364-369.

[56] A.R. Kallianpur, S.A. Lee, Y.T. Gao, W. Lu, Y. Zheng, Z.X. Ruan, Q. Dai, K. Gu, X.O. Shu, W. Zheng, Breast Cancer Res. Treat. 107 (2008) 123-132.

[57] L.M. Ferrucci, A.J. Cross, B.I. Graubard, L.A. Brinton, C.A. McCarty, R.G. Ziegler, X. Ma S.T. Mayne, R. Sinha, Br. J. Cancer 101 (2009) 178-184.

[58] D.R. Richardson, D.S. Kalinowski, S. Lau, P.J. Jansson, D.B. Lovejoy, Biochim. Biophys. Acta 1790 (2009) 702-717.

[59] J.L. Heath, J.M. Weiss, C.P. Lavau, D.S. Wechsler, Nutrients 5 (2013) 2836-2859.

[60] S.V. Torti, F.M. Torti, Crit. Rev. Oncog. 18 (2013) 435-448.

[61] N. Milman, M. Kirchhoff, Ann. Hematol. 64 (1992) 22-27.

[62] E.C. Theil, J. Nutr. 141 (2011) 724S-728S.

[63] S.V. Torti, F.M. Torti, Cancer Res. 71 (2011) 1511-1514.

[64] T.E. Galesloot, S.H. Vermeulen, A.J. Geurts-Moespot, S.M. Klaver, J.J. Kroot, D. van Tienoven, J.F. Wetzels, L.A. Kiemeney, F.C. Sweep, M. den Heijer, D.W. Swinkels, Blood 117 (2011) e218-e225.

[65] T.E. Galesloot, S. Holewijn, L.A. Kiemeney, J. de Graaf, S.H. Vermeulen, D.W. Swinkels, Arterioscler. Thromb. Vasc. Biol. 34 (2014) 446-456

[66] N. Milman, Int. J. Hematol. 63 (1996) 103-135. 